\title{
Measurement of Myocardial Wall Thickening from PET/SPECT Images: Comparison of Two Methods
}

\author{
Marissa L. Bartlett, Irene Buvat, Juan Jose Vaquero, David Mok, Vasken Dilsizian, and \\ Stephen L. Bacharach
}

\begin{abstract}
Purpose: We compared two methods for measuring myocardial wall thickening from nuclear medicine perfusion scans. The first method uses the percent change in peak activity, and the second method models a profile measured across the myocardium.

Method: Mathematical simulations of the myocardium were used. In addition, images with PET or SPECT resolution were created from real MR images. Known amounts of noise were then added.

Results: The percent peak thickening (\%PT) is nonlinear with true percent thickening, especially for PET resolutions [7 $\mathrm{mm}$ full width at half-maximum (FWHM)]. For the peak method, low levels of noise (10\%) introduced an error of $8 \%$ PT for PET and of $16 \%$ PT for SPECT. Additional smoothing reduced these errors. For the fitted model, at $10 \%$ noise, the error in thickening was large: $2.3 \mathrm{~mm}$ for PET and $7.8 \mathrm{~mm}$ for SPECT.

Conclusion: The fitted model works well only with good resolution and low noise (e.g., $7 \mathrm{~mm} \mathrm{FWHM}$ and $10 \%$ ). The peak method is also sensitive to noise, especially for poorer resolutions. Additional smoothing gives more reliable results for the peak method but not the fitted method. The peak method is therefore the more generally reliable, but even this method may only allow classification of myocardial thickening into broad categories.

Index Terms: Heart-Emission computed tomography-Image registration.
\end{abstract}

There is currently much interest in quantifying myocardial thickening from perfusion images of the heart. Two methods have been proposed: one that measures the change in myocardial brightness from end-diastole (ED) to end-systole (ES) (1) and another that estimates the width of a myocardial profile by modeling its shape (2). Both of these approaches are of use in obtaining an estimate of myocardial thickening, but both also have important limitations. The aim of this article is to compare these two methods and to describe the strengths and weaknesses of each.

From the Department of Nuclear Medicine, National Institutes of Health, Bethesda, MD, U.S.A. (M. L. Bartlett, D. Mok, V. Dilsizian, and S. L. Bacharach); U66 INSERM/CNRS, Paris, France (I. Buvat); and Grupo de Bioingenieria y Telemedicina, Universidad Politecnica de Madrid, Spain (J. J. Vaquero). Address correspondence and reprint requests to Dr. S. L. Bacharach at Department of Nuclear Medicine, National Institutes of Health, Bldg. 10, Rm. 1C401, Bethesda, MD 20892-1180, U.S.A.
The first method involves measuring the maximum (i.e., peak) pixel value in some section of the myocardium, at ED and again at ES. The difference between these two values is related to myocardial thickening. However, because pixel values depend on uptake as well as on myocardial width, it is not possible to estimate absolute thickening with this method. Instead, the percent thickening is calculated as the change in peak value from ED to ES divided by the peak value at ED. This method has been widely adopted for planar imaging, SPECT, and PET and is reported to give clinically useful results (3-8).

Changes in peak intensity can be used as a measure of myocardial thickening because the maximum brightness of the myocardium is related to its width via the partial volume effect (9). For widths less than about twice the full width at halfmaximum (FWHM) of the system resolution, a change in width causes a change in peak pixel value. However, the relationship between peak intensity and width is highly nonlinear, especially for 
better resolutions $(10,11)$. At a given resolution, the same thickening can result in very different changes in peak intensity (including no change at all) depending on the myocardial width. This nonlinearity becomes less marked as the resolution degrades, although only at the cost of smaller changes in peak intensity for the same changes in width.

Because of these difficulties, another approach has been proposed (2). In this method, a count profile is measured across the myocardium from endoto epicardium. This profile is fit to a model that is assumed to represent the real myocardial uptake underlying the PET or SPECT heart image. The theory is that, for a system with perfect resolution, a profile across the myocardium resembles a square wave in which the height of the square wave indicates the (uniform) value of uptake in the myocardium and nonzero values on either side of the square wave indicate the activity in the blood pool and outside the heart. A profile measured from a PET or SPECT image is thus a blurred version of this underlying square wave profile; so a blurred square wave can be fit to the measured profile to estimate the myocardial width. This method was reported by Porenta et al. (2) to estimate myocardial width reasonably well but to have large errors for thickening estimates.

In this article, these two methods are compared: the peak method and the fitted square wave model. We made use of mathematical simulations and also of gated cardiac MR images. The MR images were manually segmented and then used to create artificial PET and SPECT images. Myocardial width and thickening could be measured very accurately from the segmented MR images and compared with results from the MR-based PET and SPECT images for each of the two methods. The effects of image resolution, background, shape of profile, and noise were investigated for each of the two methods.

\section{MATERIALS AND METHODS}

Both of the proposed methods depend on constructing profiles across the myocardium. By "profile," we mean a plot of image intensity versus distance, starting in the center of the left ventricle blood pool and moving perpendicular to the heart wall to a point just outside the epicardium. The first method-the peak method-consists simply of measuring the peak value of such an activity profile, at ED and again at ES. The percent change in peak value is then calculated, i.e., (peak value at ES peak value at $E D$ )/peak value at ED.

The second method-the fitted model method-is more complicated and requires several steps. Our implementation was as follows: (a) Estimate background on each side of the myocardium as the mode of profile values $<60 \%$ of the peak (profile values extended $\sim 50 \mathrm{~mm}$ from the center of the heart); (b) set up a template of a square wave with the estimated background levels on each side; (c) convolve the template with a Gaussian of the required FWHM; (d) calculate the correlation of the blurred template with the measured profile; and (e) repeat steps b-d with a series of templates of different widths. The estimated width is the width of that template that correlates best with the measured profile.

Our implementation of the fitted model method differed slightly from that of Porenta et al. (2) in that we estimated background separately prior to fitting the measured profile. We chose this implementation because, at the high noise levels and high resolution we wished to study, we found that fitting for all the parameters simultaneously caused the fitting to occasionally fail. Porenta et al. (2) presumably did not encounter this difficulty because they smoothed to a resolution of $10.5 \mathrm{~mm}$ and also averaged time points together to reduce noise.

\section{Images}

Gated multislice MR images were collected from five men, three with coronary artery disease and two volunteers with no known cardiac disease (average age 44 years, range 24-61 years). The images were acquired on a $1.5 \mathrm{~T}$ GE Signa as short axis slices, $10 \mathrm{~mm}$ thick, with pixel size ranging from 1.37 to $1.56 \mathrm{~mm}$. A cine multislice technique was used (flip angle $=40^{\circ}$; TR, RR interval; TE, $20 \mathrm{~ms}$ ). The gating sequence was initiated at ED from an ECG signal. For all but one subject, the gated images were collected over the whole cardiac cycle $(8$ or 16 gates/cycle), and the size of the heart cavity was used to visually select the ES image. In one case, images were collected only from ED to ES (10 gates). Between 1 and 3 midventricular slices were selected in each subject, giving a total of 11 slices. Some subjects had only one slice selected, because certain of the MR slices were not of the very high quality necessary to accurately identify the myocardial edges.

Myocardial borders were hand drawn on each ED and ES MR image. Myocardial pixels were set to 100 and all other pixels to 15 (a myocardial/ background ratio typical of our clinical fluorodeoxyglucose PET studies). The myocardium was then divided into 16 equal-angle sectors, and the true width of each sector was measured using a $2 \mathrm{D}$ version of the method by Beyar et al. (12).

The MR images were also used to create artificial PET and SPECT images. The resulting PET/ SPECT images were "artificial" in the sense that the underlying activity levels were forced to be uniform. Also, no attempt was made to model the effects of scattered photons or, for the SPECT images, the effects of attenuation. However, the car- 
diac anatomy, wall thickness, cardiac motion, etc.-all the morphological features in space and time-were those of the actual subjects.

These MR-based PET and SPECT images were created in the following way: After manual segmentation, the MR image was convolved with a 2D filter to give typical PET or SPECT acquisition resolutions of 7 and $14 \mathrm{~mm}$ FWHM, respectively. If required, noise was added as described herein. To form profiles, each image was zoomed by a factor of 4 using a sinc interpolation (13), recast in polar coordinates around a manually chosen center, and divided into 16 sectors. For each sector, a profile across the myocardium was created by averaging pixel values along the width of the sector. That is, pixels at radius $r=1$ in a given sector were averaged to produce the first point in that profile, values at radius $r=2$ were averaged to produce the second point, and so on, radially outward from the center of the blood pool.

It is common for additional smoothing to be applied after image acquisition to reduce noise. Therefore, we investigated both smoothed (postreconstruction) and unsmoothed profiles derived from the PET and SPECT MR-based images. We chose a simple Gaussian filter with an FWHM wide enough to mimic the resultant resolution often obtained in clinical PET and SPECT images. Specifically, profiles were smoothed so as to add an additional $7 \mathrm{~mm}$ to the FWHM: For example, profiles from MRbased PET images were blurred with a $12.1 \mathrm{~mm}$ FWHM Gaussian, so that the final FWHM was 14 $\mathrm{mm}$ (by sum of squares). Thus, profiles from PET images had FWHMs of $7 \mathrm{~mm}$ (the acquisition resolution) and $14 \mathrm{~mm}$ (after additional smoothing), and profiles from SPECT images had FWHMs of $14 \mathrm{~mm}$ (the acquisition resolution) and $21 \mathrm{~mm}$ (after additional smoothing).

\section{Noise}

It was important that the noise added to the MRbased images represent real image noise. Consequently, images of a uniform phantom were collected by PET or SPECT scanners, as appropriate. The PET noise images were collected on a GE Advance PET scanner in $3 \mathrm{D}$ mode with a $2 \mathrm{~mm}$ pixel size, a slice thickness of $4.25 \mathrm{~mm}$, and an image resolution of $7 \mathrm{~mm}$ FWHM. The SPECT noise images were collected on an ADAC Vertex with 3.2 $\mathrm{mm}$ pixels and an image resolution of $14 \mathrm{~mm}$. Multiple slices of the phantom were collected at multiple time points, to give many realizations of the same uniform noise. Each noise image was processed as follows: (a) The whole image was resampled to $1.56 \mathrm{~mm} /$ pixel (to match MR pixel size); (b) the mean value was subtracted so that the image values varied around zero; (c) the image values were multiplied by the required factor to scale the SD to the desired noise level.

Because the phantoms were scanned and reconstructed on the clinically used PET and SPECT scanners, the characteristics of the noise (frequency components and correlation) matched those that would be observed in PET or SPECT clinical images. The additional smoothing described was applied postreconstruction in the same way as clinical images are smoothed postreconstruction, thus again preserving the appropriate noise characteristics.

We also compared the mean pixel value in the phantom to myocardial values typical in a patient: The SD of the phantom's pixel values could then be used to calculate typical patient noise levels. In PET, we found noise levels of $\sim 25-40 \%$, assuming myocardial activities of $500-1,000 \mathrm{nCi} / \mathrm{cc}$ for a single gated slice. Averaging three slices or three time points (e.g., 2) would reduce this to noise levels of 15-25\%. For SPECT, typical noise was in a similar range: $20-30 \%$ for a single gated slice. We therefore decided to study noise levels of 10,20 , and $40 \%$. For each noise level, for each of 11 slices, we created 10 noisy realizations of both ED and ES images (i.e., a total of 660 images), plus 1 noise-free version of each slice at ED and ES (22 images). This was done at both PET and SPECT resolutions.

Profiles were measured from each of the images listed and were processed both with and without additional smoothing. To avoid confusion, profiles will be referred to by the amount of noise in the image from which they were obtained.

\section{Mathematical Simulations}

As well as images, we used simple mathematical simulations to investigate the effect of different background levels and to investigate the importance of the underlying shape of the profile. The effect of background was studied by constructing myocardial profiles as square waves with nonzero values on either side of the central value. The effect of profile shape was studied by constructing "square" waves with linearly sloping sides. The slope was specified as the distance under the side, starting at half the central value, measured as a percentage of the width of the profile (FWHM). The same slope was applied to both sides of the profile. These simulated profiles were then blurred with a Gaussian, $7.5 \mathrm{~mm}$ FWHM. Based on a review of real PET short axis images, we studied background levels of $0,10,15$, 20 , and $50 \%$ of myocardial activity. Similarly, a review of the 176 profiles from the unblurred MR images suggested that typical slopes in the sides of an underlying profile were $20-40 \%$. We therefore investigated $0,10,20$, and $40 \%$ side slope. 


\section{RESULTS}

\section{Image Resolution}

To investigate the theoretical relationship between the peak of a profile and its width, we used mathematical simulations of a square wave with zero background and perpendicular sides. In Fig. 1, the profile peak is plotted against width for a number of different resolutions. Clearly, the relationship is highly nonlinear, especially for sharper resolutions. Therefore, the same percent thickening can give very different percent changes in peak, depending on the initial myocardial width. Recall that the peak method can measure only percent thickening. We will refer to the units of this calculation as percent peak thickening (\%PT). As an example, for $7 \mathrm{~mm}$ resolution, Fig. 1 shows that a thickening from 5 to $10 \mathrm{~mm}$ (100\% thickening) will be measured as $(91-60) / 60=51 \% \mathrm{PT}$, whereas the same $100 \%$ thickening from 10 to $20 \mathrm{~mm}$ will be measured as $(100-91) / 91=10 \% \mathrm{PT}$.

We used the noise-free versions of the MR-based PET and SPECT images to study the consequences of this nonlinearity in real hearts. Figure 2 plots the $\% \mathrm{PT}$ against the true percent thickening for both unsmoothed and smoothed profiles from MR-based PET and SPECT images. Linear correlation coefficients and standard errors of the estimate (SEE) are given in Table 1.

The data in Fig. 2 came from real subjects and therefore include many different myocardial widths. Because of this, the data do not follow a single curve: In fact, they are made up of several curves, each specific to the initial (or final) width of the profile. In Fig. 2a, for example, sectors with a true width of $>14 \mathrm{~mm}$ at $\mathrm{ES}$-plotted with filled symbols-clearly fall on a particular characteristic curve different from that for sectors with thinner

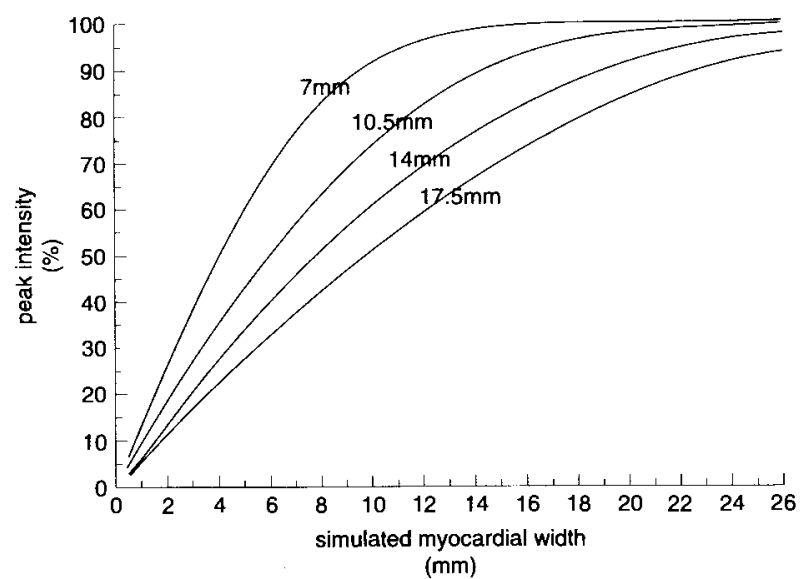

FIG. 1. Profile peak intensity versus myocardial width (simulated as the width of a square wave) for a range of resolutions that cover typical PET and SPECT resolutions. widths. The scattering of data points evident in Fig. 2 thus arises because the data represent a combination of many such curves, each corresponding to a different initial width. In particular, it should be emphasized that this spread is not the result of noise (the data contributing to this figure were noisefree). The different curves tend to overlap for smaller values of the true percent thickening and to diverge as the percent thickening increases. Thus, for three ranges of true percent thickening -50 $25 \%, 25-100 \%$, and $100-175 \%$-in Fig. $2 \mathrm{a}$, the SEEs are 4,9 , and $18 \% \mathrm{PT}$, respectively. The dependence of measured percent thickening on absolute width decreases as the resolution degrades. Thus, the spread of points is least for Fig. $2 \mathrm{~d}$, corresponding to a resolution of $21 \mathrm{~mm}$.

For the fitted model, Fig. 3 shows plots of estimated versus true values for width and thickening from noise-free MR-based images of both PET and SPECT acquisition resolution. Table 1 gives the correlation coefficient and SEEs for these plots, as well as the same results for profiles that have been subject to additional smoothing. Fig. 3 shows that the fitted model method works well with noise-free data at $7 \mathrm{~mm}$ resolution but poorly at $14 \mathrm{~mm}$ resolution: The SEEs for thickening are 1.0 and $2.7 \mathrm{~mm}$, respectively.

\section{Background}

The same partial volume effect that causes myocardial activity to be blurred into the background will also cause background activity to be blurred into the myocardium. Background activity can therefore affect the shape (and peak value) of the profile. To investigate this, profiles were simulated with different background levels for all the ED and ES widths measured from the subjects (i.e., the true widths). Again, these profiles were all noise-free. The percent thickening or thickening was calculated for backgrounds that were $0,10,15,20$, and $50 \%$ of the true myocardial intensity. For the peak method, which assumes negligible background, the percent thickening measured with no background was subtracted from the percent thickening measured with each of the other background levels to give the error due to background. The fitted model automatically estimates the background. To determine how errors in this estimate might affect thickening, the template was forced to use $15 \%$ as a background estimate while the true background was set to $0,10,15$, 20 , and $50 \%$, as described earlier.

For the peak method, we found a consistent negative bias that increased from $-10 \%$ PT for $10 \%$ background to $-30 \% \mathrm{PT}$ for $50 \%$ background (Fig. 4a). The SD associated with each background level was about the same size as the bias. The fitted 
2a

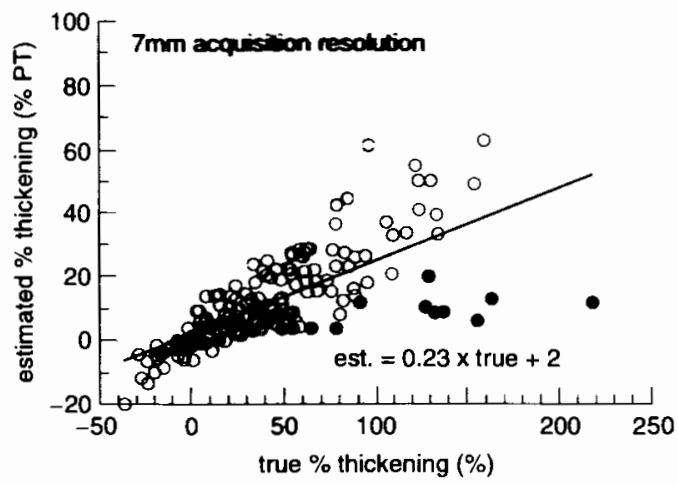

2c

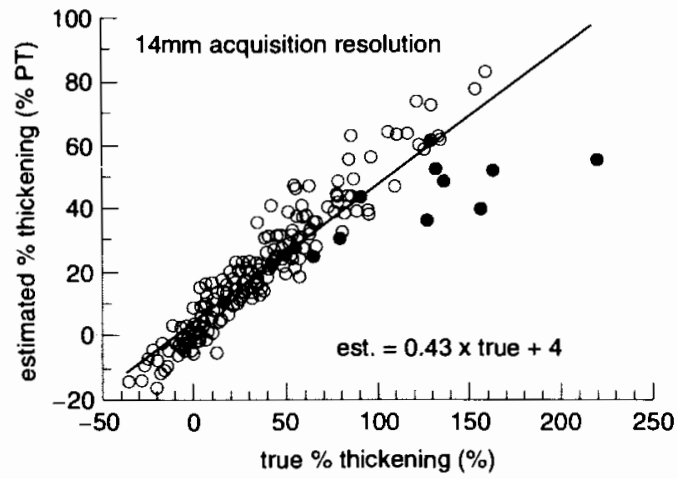

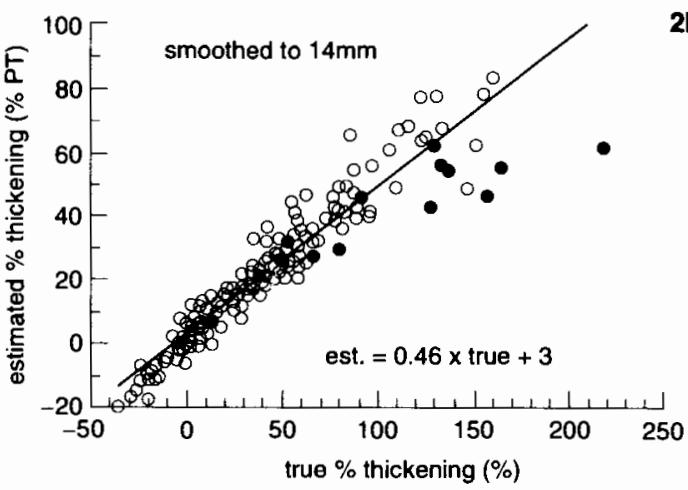

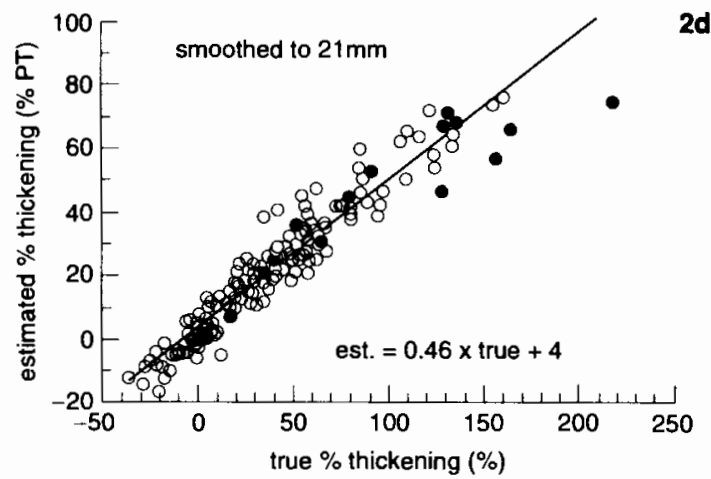

FIG. 2. Results from the peak method: estimated percent peak thickening (\%PT) versus true percent thickening from noise-free images. The filled symbols denote sectors with end-systolic width of $>14 \mathrm{~mm}$. a: PET (7 mm FWHM acquisition resolution), no additional smoothing; b: PET (7 mm FWHM acquisition resolution), smoothed to a resolution of $14 \mathrm{~mm}$ FWHM; c: SPECT (14 mm FWHM acquisition resolution), no additional smoothing; d: SPECT (14 $\mathrm{mm}$ FWHM acquisition resolution), smoothed to a resolution of $21 \mathrm{~mm}$ FWHM.

model (Fig. 4b) seems to be quite insensitive to errors in estimating background. Even in the extreme case in which the model was artificially forced to assume a $15 \%$ background while the true background was $50 \%$, the error in thickening was only $1.1 \pm 1.2 \mathrm{~mm}$. More realistically, when applied to the MR-based images with $10 \%$ noise, the algorithm estimated background to within $\pm 5 \%$. From Fig. $4 \mathrm{~b}$, this estimate of background would result in no significant bias in thickening and an SD of $\sim 0.7 \mathrm{~mm}$.

\section{Shape of Profile}

The fitted model assumes that the underlying shape of a myocardial profile is a true square wave. However, this may not in general be true: For example, tracer uptake may vary from endo- to epicardium. The peak method makes no assumption regarding profile shape, but it may be affected if the underlying shape is not consistent between profiles. To investigate this, we created simulated profiles with sloping sides for all the ED and ES widths

TABLE 1. Performance of two methods for measuring wall thickening in noise-free images

\begin{tabular}{|c|c|c|c|c|c|c|c|}
\hline & & & & \multicolumn{4}{|c|}{ Fitted model } \\
\hline & & \multicolumn{2}{|c|}{ Peak method-thickening } & \multicolumn{2}{|c|}{ Width } & \multicolumn{2}{|c|}{ Thickening } \\
\hline & & Cor. Coeff. & SEE (\%PT) & Cor. Coeff. & $\operatorname{SEE}(\mathrm{mm})$ & Cor. Coeff. & SEE (mm) \\
\hline \multicolumn{8}{|l|}{ PET } \\
\hline Acquisition resolution & $7 \mathrm{~mm}$ & 0.74 & 9.4 & 0.95 & 1.0 & 0.96 & 1.0 \\
\hline Added smoothing & $14 \mathrm{~mm}$ & 0.95 & 7.2 & 0.85 & 2.0 & 0.78 & 2.6 \\
\hline \multicolumn{8}{|l|}{ SPECT } \\
\hline Acquisition resolution & $14 \mathrm{~mm}$ & 0.92 & 8.1 & 0.68 & 2.2 & 0.67 & 2.7 \\
\hline Added smoothing & $21 \mathrm{~mm}$ & 0.96 & 6.3 & 0.10 & 2.5 & 0.32 & 2.8 \\
\hline
\end{tabular}

Estimates from noise-free MR-based images versus true values: linear correlation coefficients and standard errors of the estimate (SEE) for the two methods, for PET and SPECT acquisition resolutions, with and without additional smoothing. 
$3 \mathbf{a}$
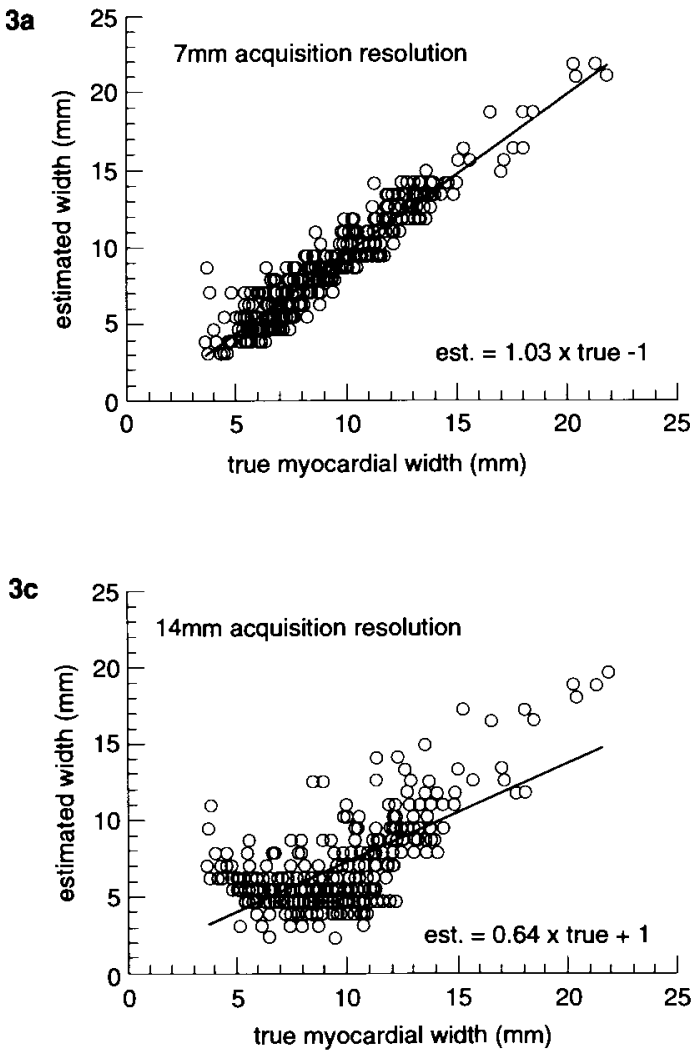

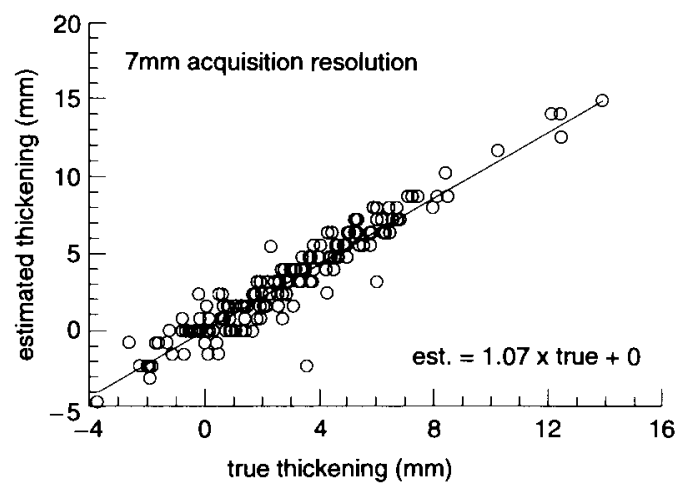

3b

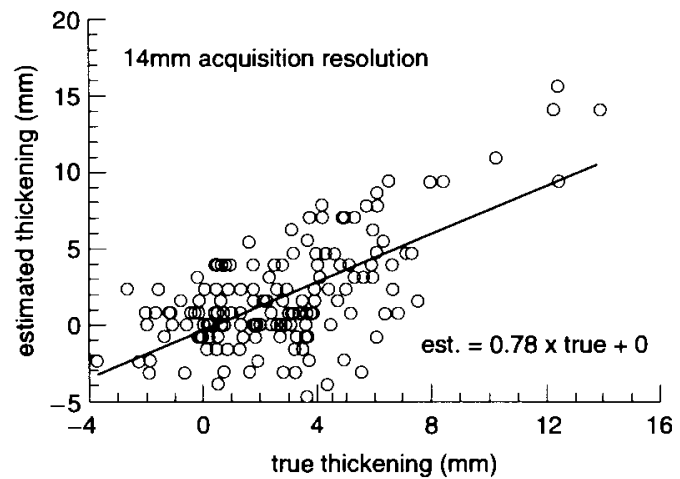

FIG. 3. Results from the fitted model method: estimated versus true values of width and thickening from noise-free images. a: Estimates of width for PET ( $7 \mathrm{~mm}$ FWHM acquisition resolution) with no additional smoothing; $\boldsymbol{b}$ : estimates of thickening for PET ( $7 \mathrm{~mm}$ FWHM acquisition resolution) with no additional smoothing; c: estimates of width for SPECT (14 mm FWHM acquisition resolution) with no additional smoothing; d: estimates of thickening for SPECT (14 mm FWHM acquisition resolution) with no additional smoothing.

measured from the subjects. For each side slope, the percent thickening or thickening was calculated and compared with the result for perpendicular sides. Neither method was strongly influenced by the presence of sloping sides. For the peak method, we found a bias of $<2 \% \mathrm{PT}$ and a variability that increased from 7 to $10 \% \mathrm{PT}$ as the side slope increased from 10 to $40 \%$. For the fitted model, the results were very similar for all side slopes: a bias of $-0.3 \mathrm{~mm}$ or less and a variability of $\sim 0.5 \mathrm{~mm}$.
$4 a$

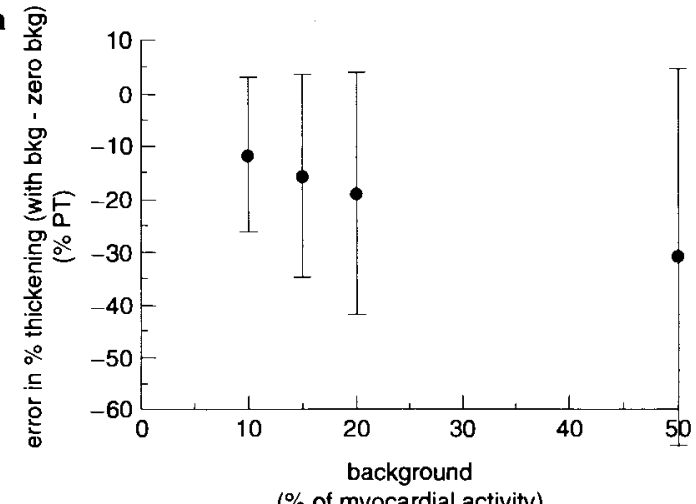

(\% of myocardial activity)
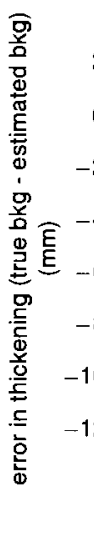

$4 b$

FIG. 4. The effect of background activity on the measurement of percent thickening. a: Peak method: change in measured percent peak thickening (\%PT) versus background (bkg) activity (as a percentage of myocardial activity); $\mathbf{b}$ : fitted model: change in measured thickening versus background activity (as a percentage of myocardial activity). For the fitted model, the model was forced to use a background of $15 \%$. 


\section{Noise}

To investigate the effects of noise, we compared the (percent) thickening measured from the noisefree MR-based images with that measured from noisy versions of the same images. Table 2 gives the SEEs of these results (note the SEE units are different for the two methods: \%PT for the peak method and $\mathrm{mm}$ for the square wave fitted model). This table gives information about the expected uncertainty in (percent) thickening for a known level of image noise.

For the peak method, comparing PET acquisition resolution $(7 \mathrm{~mm})$ with SPECT acquisition resolution $(14 \mathrm{~mm})$ for profiles that were not subject to additional smoothing, we found that the poorer acquisition resolution resulted in a greater sensitivity to noise. At $10 \%$ noise, for example, the SEE for unsmoothed profiles was 8 and $16 \% \mathrm{PT}$ for PET and SPECT, respectively. Table 2 also indicates the effect of extra smoothing applied to the measured profiles. Smoothing decreased the SEEs for lower noise: e.g., at $10 \%$ noise, SEE is $8 \% \mathrm{PT}$ for unsmoothed PET profiles compared with $6 \% \mathrm{PT}$ for smoothed PET profiles; for SPECT, the same comparison is 16 and $12 \%$ PT. For higher noise, however, this behavior changes. At $20 \%$ noise, there is less difference in SEE, comparing unsmoothed and smoothed profiles, and for $40 \%$ noise, smoothing actually worsens the SEE: for PET profiles, 25 and $83 \% \mathrm{PT}$, unsmoothed and smoothed, respectively.

For the fitted method, poorer resolution markedly increased the SEE due to noise. For $10 \%$ noise, the spread of thickening values is $2.3 \mathrm{~mm}$ for $7 \mathrm{~mm}$ FWHM, $7.8 \mathrm{~mm}$ for $14 \mathrm{~mm}$ FWHM, and 9.6 $\mathrm{mm}$ for $21 \mathrm{~mm}$ FWHM.

\section{DISCUSSION}

\section{Image Resolution}

The most important characteristic of the peak method is that peak brightness is not linear with myocardial width (Fig. 1). For a given resolution, thickening of a thin ED wall will cause a relatively large change in peak intensity, whereas the same thickening of a thick ED wall will cause only a small change in peak intensity. This relationship leads to the spread of percent thickening values seen in Fig. 2 , because each absolute myocardial width generates a different curve of percent thickening values. For example, ES widths of $>14 \mathrm{~mm}$-plotted as filled symbols in Fig. 2-clearly lie on a separate curve from other smaller widths. The relationships in Fig. 1 also explain the decrease in spread of percent thickening in Fig. $2 \mathrm{c}$ and $\mathrm{d}$ : Poorer resolution causes the curve of peak versus width to become more linear albeit with a lower slope. The greater linearity means that, for a given percent thickening, the \%PT at small ED widths will be nearly the same as the \%PT at larger ED widths. As discussed later, however, the lower slope also means that \%PT will be more sensitive to noise.

With the fitted model method, both width and absolute thickening can be estimated, and both should exhibit a linear relationship with the true values, with a slope of close to 1 (Fig. 3). However, the fitted model method works well only for good resolution images, on the order of $7 \mathrm{~mm}$ FWHM. This is presumably because, as the resolution degrades, a given change in width will make less and less difference to the final shape of the blurred profile. Hence, the fitted method will have more and more difficulty estimating the width accurately.

The results quoted here assume a constant image resolution. In fact, resolution varies across a PET or SPECT image, increasing the variability in results somewhat for both methods. If the variation in resolution is known, for a particular imaging system, the effect could be estimated from the results for two different resolutions given in Table 1 .

\section{Background}

The fitted model method automatically estimates background, whereas the peak method effectively assumes that background is negligible. As a result, the peak method is sensitive to background: A fixed

TABLE 2. Effect of noise on performance of two methods for measuring wall thickening

\begin{tabular}{|c|c|c|c|c|c|c|c|c|c|c|}
\hline & & & & & \multicolumn{6}{|c|}{ Fitted model } \\
\hline & & \multicolumn{3}{|c|}{ Peak method (\%PT) } & \multicolumn{3}{|c|}{ Width (mm) } & \multicolumn{3}{|c|}{ Thickening (mm) } \\
\hline & & $10 \%$ & $20 \%$ & $40 \%$ & $10 \%$ & $20 \%$ & $40 \%$ & $10 \%$ & $20 \%$ & $40 \%$ \\
\hline \multicolumn{11}{|l|}{ PET } \\
\hline Acquisition resolution & $7 \mathrm{~mm}$ & 8 & 15 & 25 & 1.6 & 4.1 & 8.4 & 2.3 & 5.8 & 11.5 \\
\hline Added smoothing & $14 \mathrm{~mm}$ & 6 & 15 & 83 & 4.2 & 7.2 & 9.5 & 5.8 & 10.0 & 13.4 \\
\hline \multicolumn{11}{|l|}{ SPECT } \\
\hline Acquisition resolution & $14 \mathrm{~mm}$ & 16 & 29 & 42 & 5.6 & 9.3 & 11.6 & 7.8 & 12.1 & 16.0 \\
\hline Added smoothing & $21 \mathrm{~mm}$ & 12 & 24 & 96 & 6.9 & 10.0 & 11.5 & 9.6 & 13.6 & 16.1 \\
\hline
\end{tabular}

Estimates from noisy images versus estimates from noise-free images: standard error of the estimate (SEE) for different noise levels $(10 \%, 20 \%, 40 \%)$, for PET and SPECT acquisition resolutions, with and without additional smoothing. Note that the units of the SEE are different for the two methods: \% peak thickening (\%PT) for the peak method and mm for the square wave fitted model. 
background causes variability and bias in the \%PT (Fig. 4), and variations in background will add extra variability. In contrast, the fitted method is relatively insensitive to background.

\section{Shape of Profile}

The effects of variations in tracer uptake were investigated by considering variations in the shape of the underlying profile. We found a small increase in variability in both techniques: $<10 \% \mathrm{PT}$ for the peak method and even smaller for the fitted method. The latter result agrees with the findings of Porenta et al. (2) who applied the fitted model to short axis and to transaxial PET images and found equally good results for both. It is perhaps surprising that the peak method is affected at all by changes in shape since this method does not make any assumptions about the exact shape of the profile. However, the peak method does assume that the underlying shape will be consistent for all profiles. For the same resolution, a sector in which the activity varies slowly up to its maximum may register a greater drop in maximum activity than a sector in which the activity profile increases rapidly.

\section{Noise}

For the peak method, a noise level of $10 \%$ gives rise to a larger variability for SPECT acquisition resolutions (16\% PT) than for PET acquisition resolutions $(8 \% \mathrm{PT})$. This result arises because worsening resolution decreases the slope of the relationship between peak intensity and myocardial width (Fig. 1). Thus, percent thickening is calculated with smaller (peak) values that are more sensitive to noise. Postacquisition smoothing can be used to reduce noise. However, this will also cause the slope between peak and width to decrease, causing an increased sensitivity to noise. The net result is then a trade-off between the increased sensitivity to noise and a lower effective noise level. From Table 2 , it can be seen that this trade-off is favorable for $10 \%$ noise, with additional smoothing improving the SEE. At $40 \%$ noise, however, the same trade-off is unfavorable (Table 2): The reduction in noise level now has a smaller effect than the increased sensitivity to noise.

Except under the most favorable circumstances ( $7 \mathrm{~mm}$ FWHM resolution, 10\% noise), the fitted model has large associated variability. As already discussed, its performance degrades sharply with poorer resolutions. This means that the net effect of additional smoothing is to increase the fitted model's SEE for all noise levels.

\section{Overall Variability}

Errors due to system resolution, background, profile shape, and noise level add (by sum of squares) to give the total error for each method. However, errors in any variable are most meaningful in terms of the range of values associated with that variable. In the five subjects we studied, we observed a total range of $100 \% \mathrm{PT}(-20-80 \% \mathrm{PT})$ for the peak method and a total range of $20 \mathrm{~mm}$ thickening $(-5-15 \mathrm{~mm})$ for the fitted model.

Using the results presented in this article, the total error in the peak method for $10 \%$ noise is about one-quarter of the percent thickening range. This increases to about one-third of the range for $20 \%$ noise. These total uncertainties apply to profiles with postacquisition smoothing, at both PET and SPECT resolutions. The fitted model, for $7 \mathrm{~mm}$ FWHM resolution, has a total error of about onesixth of the range for $10 \%$ noise and about one-third of the range for $20 \%$ noise. Further, the fitted model method performs very poorly for $14 \mathrm{~mm}$ FWHM resolution: The total error is almost half the range of thickening even for $10 \%$ noise.

The peak method requires either a poor system resolution or additional smoothing to make the assumption of linearity valid. This will increase sensitivity to background activity and also to noise. However, except at high initial noise levels, the reduction in noise due to smoothing will lead to a net improvement. At best, the peak method has an error of 25-30\% of its range. Thus, the peak method would appear to be useful for identifying a few broad ranges of thickening, but may not be sufficiently reliable for accurate quantification.

It is striking that the fitted model is comparable with the peak method only for low noise and good resolution: In all other circumstances, the peak method has a lower error. These results are in agreement with Porenta et al. (2) who found large errors in thickening from images with a resolution of $10.5 \mathrm{~mm}$ FWHM. It should also be noted that other effects not considered in this article-scatter and (for SPECT) attenuation-will degrade the image contrast, effectively increasing the noise level. This may further emphasize the relative robustness of the peak method.

\section{CONCLUSION}

Two methods of estimating myocardial thickening were compared. The peak method can estimate only percent thickening, whereas the fitted model method gives estimates of both width and absolute thickening. However, the fitted model works well only with good resolution and low noise. The peak method, therefore, is the more generally reliable of the two.

Unfortunately, even the peak method has associated errors that constitute a large percentage of the range of expected values. Therefore, the peak method may be useful for classifying myocardial 
thickening into a few broad categories, but has too large a variance to produce reliable quantitative measurements.

\section{REFERENCES}

1. Yamashita K, Tamaki N, Yonekura Y, et al. Quantitative analysis of regional wall motion by gated myocardial positron emission tomography: validation and comparison with left ventriculography. $J$ Nucl Med 1989;30:1775-86.

2. Porenta G, Kuhle W, Sinha S, et al. Parameter estimation of cardiac geometry by ECG-gated PET imaging: validation using magnetic resonance imaging and echocardiography. $J$ Nucl Med 1995;36:1123-9.

3. Yamashita K, Tamaki N, Yonekura Y, et al. Regional wall thickening of left ventricle evaluated by gated positron emission tomography in relation to myocardial perfusion and glucose metabolism. J Nucl Med 1991;32:679-85.

4. Mochizuki T, Murase K, Fujiwara Y, Tanada S, Hamamoto $\mathrm{K}$, Tauxe WN. Assessment of systolic thickening with thallium-201 ECG-gated single-photon emission computed tomography: a parameter for local left ventricular function. $J$ Nucl Med 1991;32:1496-500.

5. Cooke CD, Garcia EV, Folks RD, Ziffer JA. Myocardial thickening and phase analysis from Tc-99m sestamibi multiple gated SPECT: development of normal limits. $J$ Nucl Med 1992;33:926-7.

6. Marzullo P, Marcassa C, Sambuceti G, Parodi O, L'Abbate A. The clinical usefulness of electrocardiogram-gated Tc$99 \mathrm{~m}$ methoxy-isobutyl-isonitrile images in the detection of basal wall motion abnormalities and reversibility of stress induced perfusion defects. Int J Card Imag 1992;8:131-41.

7. Takeda T, Toyama H, Ishikawa N, et al. Quantitative phase analysis of myocardial wall thickening by technetium-99m 2-methoxy-isobutyl-isonitrile SPECT. Ann Nucl Med 1992; 6:69-78.

8. Pace L, Betocchi S, Piscione F, Mangoni di Santo Stefano ML, Chiariello M, Salvatore M. Evaluation of myocardial perfusion and function by technetium-99m methoxy isobutyl isonitrile before and after percutaneous transluminal coronary angioplasty. Preliminary results. Clin NuCl Med 1993;18: 286-90.

9. Hoffman EJ, Huang S-C, Phelps ME. Quantitation in positron emission computed tomography: 1. Effect of object size. J Comput Assist Tomogr 1979;3:299-308.

10. Mok DY, Bartlett ML, Bacharach SL, et al. Can partial volume effects be used to measure myocardial thickness and thickening? In: Computers in cardiology. Los Alamitos: IEEE Computer Society Press, 1992:195-8.

11. Bartlett ML, Bacharach SL, Voipio-Pulkki L-M, Dilsizian V. Artifactual inhomogeneities in myocardial PET and SPECT scans in normal subjects. $J$ Nucl Med 1995;36:18895.

12. Beyar R, Shapiro EP, Graves WL, et al. Quantification and validation of left ventricular wall thickening by a threedimensional volume element magnetic resonance imaging approach. Circulation 1990;81:297-307.

13. Smith T, Smith MS, Nichols ST. Efficient sinc function interpolation technique for center padded data. IEEE Trans Acoust Speech Signal Proc 1990;38:1512-7. 